\title{
Elaborated Intrusion Theory: A Cognitive-Emotional Theory of Food Craving
}

\author{
Jon May • Jackie Andrade • David J. Kavanagh • \\ Marion Hetherington
}

Published online: 28 February 2012

(C) Springer Science+Business Media, LLC 2012

\begin{abstract}
A clear understanding of the cognitive-emotional processes underpinning desires to overconsume foods and adopt sedentary lifestyles can inform the development of more effective interventions to promote healthy eating and physical activity. The Elaborated Intrusion Theory of Desires offers a framework that can help in this endeavor through its emphases on the roles of intrusive thoughts and elaboration of multisensory imagery. There is now substantial evidence that tasks that compete for limited working memory resources with foodrelated imagery can reduce desires to eat that food, and that positive imagery can promote functional behavior. Meditation mindfulness can also short-circuit elaboration of dysfunctional cognition. Functional Decision Making is an approach that applies laboratory-based research on desire, to provide a motivational intervention to establish and entrench behavior changes, so healthy eating and physical activity become everyday habits.
\end{abstract}

Keywords Food cravings - Elaborated intrusion - Appetite · Sensory imagery $\cdot$ Affect regulation - Acceptance-based interventions · Functional decision making · Behavior change

J. May $(\bowtie) \cdot J$. Andrade

School of Psychology, University of Plymouth,

Drake Circus, Plymouth PL4 8AA, UK

e-mail: jon.may@plymouth.ac.uk

J. Andrade

e-mail: jackie.andrade@plymouth.ac.uk

\section{J. Kavanagh}

Institute of Health \& Biomedical Innovation and School of Psychology \& Counselling, Queensland University of Technology, Brisbane, Australia

e-mail: david.kavanagh@qut.edu.au

\section{Hetherington}

Institute of Psychological Sciences, University of Leeds, Leeds, UK

e-mail: m.hetherington@leeds.ac.uk

\section{Introduction}

Despite a sophisticated homeostatic physiological system to signal hunger and satiety, our evolutionary history of periodic scarcity and abundance has endowed humans with a predisposition to overconsume when food is easily available, to endure periods of scarcity [1], and an appetite system that is asymmetrical [2]. When environments provide abundant, cheap, and highly palatable food sources, this leads to persistent overeating, and thus obesity. Alongside hormonal cues for hunger (ghrelin [3]) and satiety [cholecystokinin (CCK), peptide YY (PYY), and leptin] [4]), humans exert conscious control over food intake. Impairment of conscious memory in amnesia can have profound effects on eating [5,6]. Every episode of eating is potentially subject to the decisions to seek out food, start eating, and stop eating. If effective ways can be developed to influence this decision making, we may be able to offer strategies to support individuals whose food intake has become dysregulated.

\section{Deciding to Eat}

Human decision making has long been recognized as being only approximately rational [7]. While we can engage in careful and logical thought, much of our day-to-day decision making is not based upon deduction and inference but is heuristic, influenced by mood and proximal aspects of situations. Decision making appears to involve two quasiindependent systems, a faster, automatic "System 1" and slower, controlled "System 2" [8]. System 1 is an evolutionarily old form of cognition, which aims to reach approximately correct answers rapidly on the basis of prior experiences and ease of computation. System 2 is a newer 
form of cognition, based upon our abstract capacity for language, which can override System 1 when contexts allow and motivation enables.

Within this framework, hormonal cues to appetite and satiety influence System 1 decision making by directing attention toward food cues in the environment, and through physiological precursors to eating such as ghrelin secretion and borborygmi. Working outside conscious awareness, such processes increase the cognitive availability of foodand eating-related stimuli, making cues easier to process and more available for recall [9]. If food is to hand this might allow someone to graze without even realizing that they are consuming a significant amount of energy, so-called "mindless" eating [10]. If immediate consumption is not possible, the increasing availability of food-related thoughts eventually leads to an intrusion into consciousness of an apparently spontaneous thought about eating.

\section{Elaborated Intrusion Theory}

This distinction between unconscious precursors and conscious thought underlies a recent theory of craving that sees the conscious aspects of desire for addictive substances as part of a continuum of appetitive thought, including desire to eat. The Elaborated Intrusion Theory of Desire (EI theory) [11] describes the conscious experience of craving as a cycle of mental elaboration of an initial intrusive thought. Encounters with cues or cognitive, emotional, or physiological associations to food can trigger intrusive images or thoughts, which may feel spontaneous, as their elicitation is unconscious. These thoughts are initially pleasurable, as they share some cognitive properties of the actual desired object or activity. This motivates the individual to elaborate the thought, by retrieving cognitive associations and creating mental imagery of the target. Imagery sustains motivation because it is emotionally charged [12, 13]. If other cognitive demands allow, this imagery can take over the individual's chain of thought, dominating experience. If the desire cannot be satisfied, the experience becomes unpleasant, as the conflict between the current state of not having and the goal of having becomes more and more apparent. Recent data obtained using magnetoencephalography support the argument that this conflict state, or "mental contrasting" involves mental imagery [14]. When deprivation is high and cannot be assuaged, the ensuing worsening of mood stimulates increasingly vivid imagery, in a downward spiral with spikes of briefly pleasurable imagery leading to enhanced awareness of deficit and worsening mood. Eventually, the individual must find a way of breaking the cycle, by seeking out the target and satisfying the desire, or by cognitively breaking into the cycle through distraction or redirection of their thoughts. The latter route, while not leading to immediate consumption, leaves original triggers of the episode unaffected, increasing the likelihood of other episodes in the near future.

\section{Craving and Consumption}

In the EI theory, craving is an important target for tackling overconsumption because the cognitive and emotional processes that constitute craving drive this motivated behavior. Craving appears to have an important role in the development of obesity [15], and there is evidence that craving is associated with both binge eating [16] and early dropout from weight-loss programs [17]. Recent evidence from brain imaging studies suggests that obese individuals who experience strong cravings share with drug addicts dysregulated brain reward systems, specifically dopaminergic function [18]. Expectations and anticipations of reward from food (or drugs) have stronger impact than the neurophysiological response, maintaining overconsumption. As with drug cravings, which can occur in the absence of physiological withdrawal, craving for food can be dissociated from physiological states such as hunger [19] and nutritional status [20]. Moreover, periods of dieting or of abstinence from specific drugs are associated with decreased activity in frontal brain regions involved in salience attribution and impulse control, increasing vulnerability to cravings and intrusive thoughts [18].

The roles of intrusive thoughts and mental imagery within the EI model of desire have been supported by selfreports in surveys. Four triggers that were typically thought to have caused food cravings [21] were that the person "suddenly thought about it," "felt . . . discomfort," "imagined the taste/smell of it," or "pictured myself having it." Self-identified chocolate addicts indicated that cravings were rarely elicited by hunger and more often by mood or by external cues that reminded them of chocolate [22]. Appetite played a relatively minor role for appetite in problematic eating, with "I felt hungry" frequently triggering craving for meals, but doing so significantly less commonly for sweet or savory take-away foods [23]. Spontaneous thoughts, imagined smell or taste, and visual imagery were most often endorsed, and auditory stimuli joined sights and smells as moderately common triggers of meal-related craving.

Overall, cravers report their thoughts about the desired target as spontaneous, involving olfactory, gustatory, or visual imagery. They do not always report that their craving occurs in habitual usage situations, but since we know that situational cues are reliable triggers of craving, this suggests a relatively automatic ("System 1") process that is not necessarily open to retrospective awareness may underlie the initiation of craving. 


\section{Sensory Imagery in Craving}

Sensory imagery is a consistent feature of subjective reports of cravings for food [24], tobacco, or alcohol [25, 26] and even for playing sport [27•]. Elaborating sensory imagery not only extends episodes of craving, it makes craving more intense, as the images become better articulated and more vivid. So, people who are trying to address alcohol problems typically have sensory imagery during their strongest craving episodes, with their imagery combining more than two senses on average [25]. Similarly, intensity of craving is significantly associated with visual, gustatory, and olfactory imagery, and when participants were asked to imagine eating their favorite food, a multivariate linear regression found that hunger, visual imagery, and overall image vividness provided unique predictive variance [23]. Within participants, our research found that the only variable predicting strength of craving was the vividness of mental imagery [27•].

More vivid imagery of smoking scenarios is associated with stronger urges to smoke [28], and alcohol craving can be induced by asking clients to imagine entering their favorite bar, ordering, holding, and tasting a cold, refreshing glass of their favorite beer: guided imagery such as this can be more successful at inducing cravings in the laboratory than actually showing people a real drink [29]. Imagery of coffee drinking produces a strong craving for coffee, and as with food cravings, visual, gustatory, and olfactory senses are most commonly involved [30].

There may be situations where imagery does not increase craving. Imagining eating a chocolate sweet 30 times reduces consumption for chocolate on a subsequent "taste test," more than imagining repeatedly putting a coin in a slot machine [31•]. Thus, in excess, imagery can cause satiation rather than craving. This effect may be due to habituation, with the repeated image losing its emotive power over the course of the task, just as actually eating 30 chocolate sweets produces sensory-specific satiety [32]. Alternatively, the effect may occur through imaginary completion of the goal to eat chocolates, as uncompleted goals remain hyperaccessible [33] and therefore liable to trigger intrusive thoughts and craving [9]. While repeated imagery can reduce consumption in the laboratory, it is difficult to see how this technique could easily be applied in the natural environment, as the person may run an increased risk of consumption during early trials [34].

\section{Concurrent Visuospatial Tasks}

The importance of sensory images and experiences in craving is also supported by the impact of craving on concurrent tasks. While people with high trait craving for chocolate show impaired performance from heightened craving on reaction times and verbal working memory [35], more reliable effects on concurrent tasks are obtained when they involve visuospatial (eg, Corsi Blocks task) rather than verbal working memory (eg, digit span) [36•].

Interference from elaborative craving on concurrent tasks suggests that such tasks may be used to disrupt craving [21]. Consistent with this idea, several studies have found that manipulating visual imagery or loading visuospatial working memory reduces the strength of food cravings. These studies tested hungry participants craving food in general or participants craving chocolate, which is associated with rather specific cravings that cannot be satisfied by nutritionally similar foods $[22,37]$. The research has shown that craving can be reduced by visual or olfactory images of unrelated scenes [30,38], by watching a rapidly changing visual display [39], and by performing tasks that require spatiomotor control as well as visual working memory processes, such as modeling plasticine or clay, making side-toside eye movements, or tapping a pattern on a keyboard $[40 \cdot 41]$.

Consistent findings also come from research on delayed gratification, where asking participants to create "vivid fantasies" [42] of a different substance is a powerful way of increasing the time for which they will wait for a reward. Asking preschoolers to think about the crunchiness and saltiness of pretzels enabled them to resist the temptation of a marshmallow for an extra $17 \mathrm{~min}$, compared with thinking about marshmallows or thinking about less obviously pleasurable aspects of pretzels such as how long they are [43]. Instructing participants to imagine chocolate in "a strange and extraordinary way in a context unrelated to consumption" reduced responses to chocolate on an Implicit Attitudes Test compared with imagining "as clearly and concretely as possible how eating the chocolate would taste and feel" [44]. This supports the EI theory contention that there is a cyclical relationship between consumption-related elaboration (including imagery) and increased availability of associated representations, which in turn increases the likelihood of intrusive thoughts [9].

\section{Intrusive Thoughts}

An alternative to weakening the elaborative cycle is to prevent the cycle starting in response to the intrusive thought, or by lowering the probability of the intrusive thoughts occurring in the first place. This may be the strategy that most often occurs to people who find themselves experiencing unwanted thoughts about foods that they are trying to avoid. Trying not to think about something, however, has been shown to be counterproductive, in that it leads people to self-monitor their thoughts to check that 
they are not thinking about the forbidden topic, only to realize that this reminds them about it. Equally, trying to distract oneself by always thinking about a certain topic whenever you find yourself thinking about food leads to that topic also being associated with food, creating yet another cue to cravings. People who are instructed to suppress thoughts about chocolate for 5 min subsequently eat more chocolate in a "taste test" than a control group who had to simply verbalize their thoughts, without any requirement not to think about chocolate [45•], and food thought suppression has also been shown to predict binge eating, food cravings, and other eating-disordered symptoms [46]. Furthermore, some individuals - either through genetic predisposition or changes to neurochemistry from repeated dieting-overeating cycles-are more vulnerable to food craving and to intrusive thoughts about food [47]: interventions need to accommodate the likelihood that such individuals are likely to have frequent intrusive thoughts even if they are not attempting to suppress them.

\section{Acceptance-Based Interventions}

It may be more beneficial to let intrusions occur, but to change the way people respond to them. By providing information that spontaneous thoughts do not need a behavioral response, people can learn to accept them, letting them pass without further elaboration. This is one of the ideas behind acceptance-based therapies, or mindfulness training, which are increasingly used in psychological treatments, especially for disorders where intrusive thoughts are known to play a role, such as depression, obsessive compulsive disorder, and anxiety [48, 49]. Acceptance-based interventions have been shown to help in managing food cravings [50] and maintaining weight loss [51]. The cognitive states engendered by such interventions are also predictive of positive outcomes (eg, the Food Craving Acceptance and Action Questionnaire predicts weight reduction in women enrolled in weight loss treatment) $[52,53]$.

Components of mindfulness include developing focus on present experience, so that thought is dominated by a range of sensory inputs rather than following well-learned paths of association and secondary cognitive processing. For example, paying closer attention to food as it is eaten produces greater levels of satiation and reduced food intake compared to directing focus away from the eaten food [52, 53], and remembering what was eaten at a previous meal suppresses intake at a later snack [54].

Body scanning and breath focus routines are often used to shift attention to internal bodily sensations. In hungry volunteers, focusing upon breath increases cravings and thoughts about food [55•], because the bodily sensations associated with breathing and attending to the abdominal region include those associated with hunger. In contrast, a scan incorporating the whole body can reduce the frequency of thoughts about food. However, we did not find that this whole-body scan differentially reduced craving compared with controls [55 ${ }^{\bullet}$, presumably because the working memory load of that task did not sufficiently interfere with the intensity of craving-related imagery that did occur. Related work has shown that body scanning can reduce cravings for cigarettes [56], and can reduce the frequency of thoughts about smoking as well as cigarette cravings [57, 58].

Meditation may not only achieve results via cognitive refocusing, and some potential contributors to its effect may be shared with other interventions. In fact, one rationale for the use of body scanning is that it may act in a similar way to isometric exercises, in moderating cravings by reducing stress [56]. A review of the effect of exercise on cigarette craving found that relatively small doses helped abstaining smokers manage cigarette cravings and withdrawal [57, 58], and a 15-minute walk reduced cue-elicited chocolate cravings [59]. Engaging in exercise is of course likely to be part of any recommendations made to individuals who are overweight, but this suggests that it might also play a role in reducing cravings and resultant energy intake, as well as increasing energy expenditure.

\section{Affect Regulation}

There is now substantial evidence that stress has a role in compensatory overeating [60]. Individual differences in increases in negative mood in response to stressors are significantly related to greater food consumption [61], and evidence from longitudinal studies suggests that chronic life stress may be causally linked to weight gain [62]. Shortterm stress does not alter overall intake or appetite, but stressed "emotional eaters" eat more sweet high-fat foods and a more energy-dense meal than unstressed and nonemotional eaters [63]. Similarly, self-reported stress specifically produces overeating in emotional eaters, directing food choice to high-fat sweet items (eg, biscuits, chocolate) or high-fat salty foods (eg, potato chips), and specific types of stress such as ego-threatening stressors inhibit eating in restrained eaters [64].

When people are upset, they indulge in immediate impulses to make themselves feel better, giving short-term affect regulation priority over other self-regulatory goals [65]. However, believing that one's bad mood is unchangeable eliminates the tendency to eat fattening snacks [65]. This idea is broadly consistent with the EI theory, which holds that negative emotions tend to induce a sense of generalized deprivation, which can readily be misattributed to a deprivation of a salient target (eg, a food that is commonly craved). If the person believes that their deprivation 
cannot be assuaged by a food such as chocolate, related craving is likely to be much less. The EI theory also predicts that effects of negative emotion are likely to be greater in people who are more prone to experience it, are more aware of it, or have repeatedly used food in an attempt to address negative emotions in the past. So, women (who are more likely to report negative emotions) more commonly cite them as triggers of food cravings than do men [23].

\section{Implications for Behavior Change}

Combining the evidence from these studies on craving for food and other substances, one approach to influencing decisions to eat is to acknowledge that food desires are normal, and are not in themselves unhealthy. Thoughts about food indicate a behavioral option rather than representing an inescapable need. Food desires exist in a context of other desires (eg, to feel energized or relaxed, to feel happier, or to reward oneself at the end of a hard day). Food is just one possible way of meeting those goals, and there are other ways to reach the same ends. Furthermore, some concurrent desires may be incompatible with unrestricted eating - feeling energized may be linked to feeling healthy and fit, and having less weight to carry. Maximizing healthenhancing strategies to meet valued goals may be more effective than focusing on avoidance of pleasurable eating.

Consistent with this idea, activities with immediate moodenhancing effects become a focus of behavioral change. While some benefits of improved dietary or exercise regimens can occur relatively early in an attempt (eg, emotional responses to early achievements or to endorphin release during exercise), short-term impacts on weight and fitness come in small increments that can be hidden by transitory confounds (eg, with weight, food in the alimentary tract, or degree of fluid retention related to menstrual cycles or other factors).

Competing pleasurable activities should be simple and at least as easy to do as acquiring and consuming a snack. So, a task with a positive goal that involves walking up and down stairs (eg, to look at picture of your family that is in the bedroom) may take only 2 to $3 \mathrm{~min}$, but may provide both the benefits of mild exercise [59] and interfere with elaborative mental imagery through the visual and spatiomotor processing required to complete it.

Anorexics, who regularly withstand thoughts about food and resist responding to the physical and sensory sequelae of hunger, change the way that they elaborate food thoughts so they focus on positive consequences of not eating, as much as the negative consequences of eating [66•]. Not eating in response to thoughts of food signals that they are in control, and sensations of hunger indicate that they are succeeding in their goal of weight loss. With overweight individuals, a similar remapping of elaborative thoughts onto the positive consequences of not eating may be possible, so that a thought about food is interpreted as a sign that they are succeeding in weight loss.

In this approach, individuals identify short activities that can easily be incorporated into their daily routine, as well as introducing some physical activities of longer duration. In both cases, the primary focus is on activities that are pleasurable or highly rewarding, to maximize the chance that they will be maintained, and ideally so they will also generate desire imagery. This idea capitalizes on the weightreducing impact of physical exercise [67], while potentially avoiding the potential risks of extended activities becoming too difficult to maintain, triggering increased caloric intake [68], or of energy expenditure being offset by decreased incidental activity [69]. The role that exercise can play in reducing intake may initially be more important than its impact on weight, although the two aims are concordant.

At the same time, meditation mindfulness is introduced, together with tasks that can compete with food-related cognitions, including vivid, multisensory imagery of positive feelings and sensations during and after exercise. When cravings do subsequently emerge, the individual has then acquired a repertoire of strategies to compete with food imagery and lessen its tendency to trigger elaboration or rumination.

\section{Motivation Enhancement}

These strategies can be embedded within motivation enhancement (ME), which encourages participants to consider and discuss functional behavior change, including considering the advantages of positive goals. ME has been used successfully to enhance weight loss from standard care or behavioral programs [70], although the size of the increased loss is relatively modest $(1.47 \mathrm{~kg})$. The strongest effects were obtained where diabetic women received five 45minute sessions at three monthly intervals to 12 months: however, even there, the difference in weight loss was only $1.8 \mathrm{~kg}$ at 12 and 18 months [71]. While these are positive results, the effects are modest.

The strategies suggested above are highly consistent with the content and style of ME, in helping individuals focus on their own goals, motivations, and building self-efficacy. However, they extend it by explicitly rehearsing episodic imagery about the advantages of change, past achievements, and future plans. Evidence on the importance of imagery about motivations has already been presented above: Past achievements constitute a powerful determinant of selfefficacy [72], and elaborating imagery about these recalled events maximizes their impact on confidence.

Implementation intentions (IIs), or detailed plans for change, are also explicitly integrated into the intervention. IIs are already in ME, but are unlikely to be articulated unless the person 
has committed to change: Consistent with the EI theory, we introduce a hypothetical articulation of plans that are undertaken regardless of the person's commitment. IIs are known to increase healthy eating [73]: we further augment their impact using imagery. This idea, which overlaps with the concept of imaginal or covert rehearsal, has already been shown to increase fruit consumption more than conventional forms of II, in college students who were low consumers of fruit [74•].

Consistent with the thematic inclusion of motivational approaches [71], each of these strategies is threaded through interventions and practiced by participants, so they become habitual ways to maintain behavior change.

\section{Functional Decision Making}

Our approach also explicitly extends ME into the natural environment, encouraging participants to rehearse episodic images about predicted and actual benefits, successes and coping behaviors within situations where they are tempted to engage in less functional behaviors such as overeating or inactivity. In this way, it more fully utilizes the power of positive imagery to compete with other desires, build self-efficacy and cue effective coping. The emphasis on this feature is reflected in the title given to the overall intervention-Functional Decision Making. Our key objective is to encourage participants to make decisions in their everyday lives, that will maximize the functional benefits they experience as a result, and all of the described components of the intervention serve that end.

\section{Conclusions}

Overconsumption is supported by overlearned, automatic behaviors and by conscious responses to the subjective experience of food cravings. By understanding cognitive processes linking situational and physiological triggers, intrusive thoughts, and elaborative imagery, we can develop interventions that bring automatic behavior under conscious control, and provide individuals with the mental strategies to resist cravings, to support them in making the decision not to consume. These interventions include redefining the implications of food-related thoughts, weakening cravings through brief visuospatial tasks, and strengthening positive imagery-based representations of alternatives to consumption. Within Functional Decision Making, we help people establish and entrench these strategies into their everyday lives, so that healthy lifestyles become healthy habits.

Disclosure No potential conflicts of interest relevant to this article were reported.

\section{References}

Papers of particular interest, published recently, have been highlighted as:

- Of importance

1. Levin E. Why some of us get fat and what we can do about it. J Physiol (Lond). 2007;583:425-30.

2. Blundell JE, King NA. Overconsumption as a cause of weight gain: behavioural-physiological interactions in the control of food intake (appetite). Ciba Found Symp. 1996;201:138-54.

3. Cummings DE. Ghrelin and the short- and long-term regulation of appetite and body weight. Physiol Behav. 2006;89:71-84.

4. Schwartz MW, Woods WC, Porte D, et al. Central nervous system control of food intake. Nature. 2000;404:661-71.

5. Rozin P, Dow S, Moscovitch M, Rajaram S. What causes humans to begin and end a meal? A role for memory for what has been eaten as evidenced by a study of multiple meal eating in amnesic patients. Psychol Sci. 1998;9:392-6.

6. Higgs S, Williamson AC, Rotshtein P, Humphreys GW. Sensoryspecific satiety is intact in amnesics who eat multiple meals. Psychol Sci. 2008;19:623-8.

7. Gigerenzer G, Gaissmaier W. Heuristic decision making. Annu Rev Psychol. 2011;62:451-82.

8. Evans JStBT. Dual-processing accounts of reasoning judgment and social cognition. Annu Rev Psychol. 2008;59:255-78.

9. Berry L-M, Andrade J, May J. Hunger-related intrusive thoughts reflect increased accessibility of food items. Cogn Emot. 2007;21:865-78.

10. Wansink B. Mindless eating: why we eat more than we think. Bantam Books, 2006

11. Kavanagh DJ, Andrade J, May J. Imaginary relish and exquisite torture: the elaborated intrusion theory of desire. Psychol Rev. 2005;112:446-67.

12. Bywaters M, Andrade J, Turpin G. Determinants of the vividness of visual imagery: the effects of delayed recall stimulus affect and individual differences. Memory. 2004;12:479-88.

13. Holmes EA, Mathews A. Mental imagery and emotion: a special relationship? Emotion. 2005;5:489-97.

14. Achtziger A, Fehr T, Oettingen G, et al. Strategies of intention formation are reflected in continuous MEG activity. Soc Neurosci. 2009;4:11-27.

15. Schlundt DG, Virts KL, Sbrocco T. Pope-Cordle: a sequential behavioral analysis of craving sweets in obese women. Addict Behav. 1993;18:67-80.

16. Gendall KA, Joyce PR, Sullivan PF, Bulik CM. Food cravers: characteristics of those who binge. Int $\mathrm{J}$ Eat Disord. 1998;23:353-60.

17. Sitton SC. Role of craving for carbohydrates upon completion of a protein-sparing fast. Psychol Rep. 1991;69:683-6.

18. Volkow ND, Wang GJ, Fowler JS et al. Food and drug reward: overlapping circuits in human obesity and addiction. Curr Top Behav Neurosci, in press.

19. Lafay L, Thomas F, Mennen L, et al. Gender differences in the relation between food cravings and mood in an adult community: results from the Fleurbaix Laventie Ville Santé study. Int J Eat Disord. 2001;29:195-204.

20. Pelchat ML, Schaeffer S. Dietary monotony and food cravings in young and elderly adults. Physiol Behav. 2000;68:353-9.

21. May J, Panabokke N, Andrade J, Kavanagh D. Images of desire: cognitive models of craving. Memory. 2004;12:447-61.

22. Hetherington MM, Macdiarmid JI. Chocolate addiction: a preliminary description and report of its relationship to problem eating. Appetite. 1993;21:233-46. 
23. Tiggeman M, Kemps E. The phenomenology of food cravings: the role of mental imagery. Appetite. 2005;45:305-13.

24. Harvey K, Kemps E, Tiggemann M. The nature of imagery processes underlying food cravings. Br J Heal Psychol. 2005;10:49-56.

25. Kavanagh DJ, May J, Andrade J. Tests of the elaborated intrusion theory of craving and desire: features of alcohol craving during treatment for an alcohol disorder. Br J Clin Psychol. 2009;48:241-54.

26. Statham DJ, Connor JP, Kavanagh DJ, et al. Measuring alcohol craving: development of the alcohol craving questionnaire. Addiction. 2011;106:1230-8.

27. - May J, Andrade J, Kavanagh D, Penfound L. Imagery and strength of craving for eating, drinking and playing sport. Cogn Emot. 2008;22:633-50. Common aspects of craving are shared between appetitive cravings and desires to engage in sport.

28. Tiffany ST, Drobes DJ. The development and initial validation of a questionnaire on smoking urges. Br J Addict. 1991;86:1467-76.

29. Litt MD, Cooney L. Inducing craving for alcohol in the laboratory. Alcohol Res Health. 1999;23:174-8.

30. Kemps E, Tiggemann M. Competing visual and olfactory imagery tasks suppress craving for coffee. Exp Clin Psychopharmacol. 2009; 17:43-50.

31. - Morwedge CK, Huh YE, Vosgerau J. Thought for food: imagined consumption reduces actual consumption. Science. 2010;330:1530 3. Imagining eating can mimic overeating through habituation.

32. Hetherington M, Rolls BJ, Burley VJ. The time course of sensoryspecific satiety. Appetite. 1989;12:57-68.

33. Förster J, Liberman N, Higgins ET. Accessibility from active and fulfilled goals. J Exp Soc Psychol. 2005;41:220-39.

34. Mischel W, Ebbeson EB, Raskoff-Zeiss A. Cognitive and attentional mechanisms in delay of gratification. J Pers Soc Psychol. 1972;21:204-18.

35. Kemps E, Tiggemann M, Grigg M. Food cravings consume limited cognitive resources. J Exp Psychol Appl. 2008;14:247-54.

36. - Tiggeman M, Kemps E, Parnell J. The selective impact of chocolate craving on visuospatial working memory. Appetite. 2010;55:44-8. Cravings impair performance on tasks that require visual working memory.

37. Michener W, Rozin P. Pharmacological versus sensory factors in the satiation of chocolate craving. Physiol Behav. 1994;56:419-22.

38. Kemps E, Tiggemann M. Modality-specific imagery reduces cravings for food: an application of the Elaborated Intrusion theory of desire to food craving. J Exp Psychol Appl. 2007;13:95-104.

39. Steel D, Kemps E, Tiggemann M. Effects of hunger and visuospatial interference on imagery-induced food cravings. Appetite. 2006;46:36-40.

40. • May J, Andrade J, Panabokke N, Kavanagh D. Visual imagery tasks suppress craving for cigarettes. Behav Res Ther. 2010;48:476-85. Tasks loading mental resources that are required to construct visual images can stop cigarette cravings from developing.

41. Kemps E, Tiggemann M, Woods D, Soekov B. Reduction of food cravings through concurrent visuo-spatial processing. Int $\mathrm{J}$ Eat Disord. 2004;36:31-40.

42. Metcalfe J, Mischel W. A hot/cool-system analysis of delay of gratification: dynamics of willpower. Psychol Rev. 1999;106:13-9.

43. Mischel W, Baker N. Cognitive appraisals and transformations in delay behaviour. J Pers Soc Psychol. 1975;31:254-61.

44. Hofmann W, Deutsch R, Lancaster K, Banaji MR. Cooling the heat of temptation: mental self-control and the automatic evaluation of tempting stimuli. Eur J Soc Psychol. 2009;40:117-25.

45. - Erskine JAK. Resistance can be futile: investigating behavioural rebound. Appetite. 2008;50:415-21. Trying not to think about eating led people to eat more after the constraint was lifted, showing that thought suppression is counterproductive.

46. Barnes RD, Tantleff-Dunn S. Food for thought: examining the relationship between food thought suppression and weight-related outcomes. Eat Behav. 2010;11:175-9.
47. Davis C, Curtis C, Levitan RD, et al. Evidence that 'food addiction' is a valid phenotype of obesity. Appetite. 2011;57:711-7.

48. Kabat-Zinn J. Mindfulness-based interventions in context: past present and future. Clin Psychol Sci Pract. 2003;10:144-56.

49. Teasdale JD, Segal ZV, Williams JMG, et al. Prevention of relapserecurrence in major depression by mindfulness-based cognitive therapy. J Consult Clin Psychol. 2000;68:615-23.

50. Forman EM, Hoffman KL, McGrath KB, et al. A comparison of acceptance- and control-based strategies for coping with food cravings: an analog study. Behav Res Ther. 2007;45:2372-86.

51. Forman EM, Butryn ML, Hoffman KL, Herbert JD. An open trial of an acceptance-based behavioral intervention for weight loss. Cognitive and behavioral practice special series: ethical challenges in cognitive behavioral research training and practice. 2009;16:223-5

52. Hetherington M. Cues to overeat: psychological factors influencing overconsumption. Proc Nutr Soc. 1997;66:113-23.

53. Jurascio A, Forman E, Tinko CA, et al. The development and validation of the food craving acceptance and action questionnaire: FAAQ. Eat Behav. 2011;12:182-7.

54. Higgs S. Cognitive influences on food intake: the effects of manipulating memory for recent eating. Physiol Behav. 2008;94:734-9.

55. - May J, Andrade J, Batey H et al. Less food for thought: Impact of attentional instructions on intrusive thoughts about snack foods. Appetite. 2010;17:126-37. Acceptance-based approaches reduced the frequency of spontaneous thoughts about snack foods, but did not affect craving.

56. Ussher M, Cropley M, Playle S, et al. Effect of isometric exercise and body scanning on cigarette cravings and withdrawal symptoms. Addiction. 2009;104:1251-7.

57. May J, Andrade J, Willoughby K, Brown C. An attentional control task reduces intrusive thoughts about smoking. Nicotine Tob Res. 2011

58. Taylor AH, Ussher MH, Faulkner G. The acute effects of exercise on cigarette cravings withdrawal symptoms affect and smoking behavior: a systematic review. Addiction. 2007;102:534-43.

59. Taylor AH, Oliver AJ. Acute effects of brisk walking on urges to eat chocolate, affect and responses to a stressor and chocolate cue: an experimental study. Appetite. 2007;52:155-80.

60. Greeno CG, Wing RR. Stress-induced eating. Psychol Bull. 1994;115:444-64.

61. Epel E, Lapidus R, McEwen B, Brownell K. Stress may add bite to appetite in women: a laboratory study of stress-induced cortisol and eating behaviour. Psychoneuroendocrinology. 2001;26:47-9.

62. Torres SJ, Nowson CA. Relationship between stress eating behavior and obesity. Nutrition. 2007;23:887-94.

63. Oliver G, Wardle J, Gibson EL. Stress and food choice: a laboratory study. Psychosom Med. 2000;62:853-65.

64. Wallis DJ, Hetherington MM. Emotions and eating: self-reported and experimentally induced changes in food intake under stress. Appetite. 2009;52:355-62.

65. Tice DM, Bratslavsky E, Baumeister RF. Emotional distress regulation takes precedence over impulse control: if you feel bad do it! J Pers Soc Psychol. 2001;80:53-67.

66. - Blackburn JF, Thompson AR, May J. Feeling good about being hungry: food-related thoughts in eating disorder. J Exp Psychopathol. in press. A qualitative study found that restricted eaters elaborated the positive aspects of not eating in response to thoughts about food, rather than the positive aspects of eating.

67. Thorogood A, Mottillo S, Shimony A, et al. Isolated aerobic exercise and weight loss: a systematic review and meta-analysis of randomized controlled trials. Am J Med. 2011;24:747-55.

68. Hopkins M, King NA, Blundell JE. Acute and long-term effects of exercise on appetite control: is there any benefit for weight control? Curr Opin Clin Nutr Metab Care. 2010;13:635-40.

69. Colley RC, Hills AP, King NA, Byrne NM. Exercise-induced energy expenditure: implications for exercise prescription and obesity. Patient Educ Couns. 2010;79:327-32. 
70. Armstrong MJ, Mottershead TA, Ronksley PE, et al. Motivational interviewing to improve weight loss in overweight and/or obese patients: a systematic review and meta-analysis of randomized controlled trials. Obes Rev. 2011;12:709-23.

71. West DS, DeLillo V, Bursac Z, et al. Motivational interviewing improves weight loss in women with type 2 diabetes. Diabetes Care. 2007;30:1081-7.

72. Bandura A. Self-efficacy: toward a unifying theory of behavior change. Psychol Rev. 1977;84:191-215.
73. Adriaanse MA, Vinkers CD, De Ridder DT, et al. Do implementation intentions help to eat a healthy diet? A systematic review and meta-analysis of the empirical evidence. Appetite. 2011;56:183-93.

74. - Knauper B, McCollam A, Rosen-Brown A, et al. Fruitful plans: adding targeted mental imagery to implementation intentions to increase fruit consumption. Psychol Health. 2011;26:601-17. Adding visual imagery to a behavior change method, IIs led to greater increases in fruit consumption. 